\title{
Liderando cuidados de Enfermería basados en evidencia a través de procesos sistematizados
}

\author{
Leading evidence-based nursing care through systematized \\ processes
}

\section{Liderança de cuidados de enfermagem baseadas em evidências através de processos sistemátizados}

Los gobiernos de todo el mundo apuntan a mejorar los cuidados de salud, y a optimizar sus resultados a través de iniciativas destinadas a acelerar la integración sistematizada de atención clínica basada en la evidencia. No es sorprendente entonces, que este sea el segundo número de la revista MedUNAB, dedicado a visualizar la transformación sistematizada de la profesión de enfermería a través del uso continuo de evidencia científica en la práctica clínica del día a día.

El primer número fue la edición de MedUNAB 17(3) y contó con cuatro artículos importantes enfocados en el programa de guías de mejores prácticas de la Asociación Profesional de Enfermeras y Enfermeros de Ontario (Registered Nurses' Association of Ontario, RNAO) $(1,2)$. Detalles de este programa se encuentran en la página web de la RNAO: http://rnao.ca/bpg.

En el presente número 19(2), se continúa con la socialización de diversas experiencias de la RNAO en países Latinoamericanos: Tres artículos se enfocan en metodologías sistematizadas para procesos de cambio institucional, tanto en instituciones clínicas como en universidades. En este sentido, Amalia Silva, Directora de la facultad de enfermería de la Universidad de Chile y jefe del programa Best Practice Spotlight Organization (BPSO) de la RNAO en dicha universidad, relata sus experiencias en llevar un proceso sistematizado de la malla curricular.

La Dra. Olga Lucía Cortés y la Directora del de Enfermería Aracelly Serna, y demás colegas de la Fundación
Cardioinfantil-IC (FCI-IC), cuentan su trayectoria como BPSO desde la selección de tres guías de buenas prácticas RNAO, al desarrollo de las capacidades necesarias para su implementación, el proceso de implementación, y su evaluación preliminar y continúa inclusive de la selección de indicadores.

La Directora de Enfermería Maribel Esparza-Bohórquez, y enfermeras Lina María Granados y Katherinne JoyaGuevara BPSO en Bucaramanga, comparten sus logros y desafíos en la implementación de la guía de buenas prácticas sobre valoración del riesgo y prevención de ulceras por presión en la Fundación Oftalmológica de Santander (FOSCAL).

Estas tres instituciones, una académica en Chile y dos hospitales colombianos de alto nivel de complejidad, demuestran la importancia y utilidad del uso sistematizado de procesos de cambio y el impacto de educación y práctica clínica basadas en la evidencia.

Los alcances son prometedores tanto en la acogida y adherencia al programa BPSO de la RNAO, como también a los tempranos resultados clínicos. Por ejemplo, tanto en la FCI-IC como en la FOSCAL, ha habido una mejoría significativa en la evaluación y re-valoración de riesgo con parámetros fijos. En la FCI-IC se observó una reducción en el uso de barandas lo cual es una de las recomendaciones más complejas de la Guía de Prevención de Caídas de la RNAO. Además, se observó una reducción en caídas. También se percibió una limitación a grado II del tipo de 
úlcera por presión (UPP) adquiridas durante la hospitalización. Así también aumentó la valoración en miembros inferiores, la educación y el cuidado en úlceras en pie en pacientes diabéticos.

En la FOSCAL por su lado, donde detallan su experiencia con la guía de RNAO de valoración del riesgo y prevención de úlceras por presión, se evidencia anualmente disminución gradual en la prevalencia de UPP, y estas también aquí están limitadas a grado II, con un total de 4\% pacientes que desarrollaron este tipo de UPP, pero un cero por ciento de UPP nivel III o IV. Así también se evidencia un aumento significativo en el porcentaje de valoración del riesgo, seguimiento y revaloración durante la hospitalización. La adherencia del personal al cumplimiento de las recomendaciones de la guía fue del 91 por ciento - un impresionante resultado.

Las guías de práctica clínica de la RNAO constituyen una síntesis de la mejor evidencia que existe sobre recomendaciones del cuidado en temas clínicos y de entorno laboral que responden a preguntas específicas. Están desarrolladas por paneles de expertos y basadas en revisiones sistemáticas, conjuntamente con todos los aspectos a considerar para determinar una decisión clínica. Esto incluye el riesgo, pronóstico, edad, costos, valores del paciente, y recursos de la institución, manteniendo un equilibrio entre los beneficios y el riesgo de una recomendación(3-6).

La evaluación de resultados se lleva a cabo a través del sistema de datos de los Indicadores de Calidad de la Enfermería para Reportaje y Evaluación (Nursing Quality Indicators for Reporting and Evaluación, NQuIRE), que provee indicadores de medición basados en la evidencia. Un sistema internacional de alta complejidad y beneficio para todos los BPSOs $(1,2)$.

Sin lugar a duda, la experiencia del implementar las Best Practice Guidelines (BPGs) y el trabajo en red con la RNAO a través de la designación BPSO van llevando a distintos países del mundo, y a las más de 500 instituciones afiliadas a esta designación, a una práctica clínica de enfermería basados en la evidencia. Una práctica clínica que se puede monitorear y medir, y por la cual se benefician pacientes y sus seres queridos, instituciones y sistemas de salud (3-6). Cabe decir que la presencia y liderazgo de los
BPSOs posicionan a Enfermería como una profesión de alto conocimiento, y a las enfermeras como profesionales que dan servicios de calidez y calidad.

Les invito a revisar y compartir estos artículos. Juntos tenemos la oportunidad de moldear el futuro de Enfermería y transformar nuestra profesión a través del conocimiento.

\section{Dra. Doris Grinspun RN, MSN, PhD, LLD(hon), O.ONT. Gerente General} Asociación de Enfermeras Registradas de Ontario

\section{Referencias}

1. Bajnok I, Grinspun D, Lloyd M, McConnell H. Leading quality improvement through best practice guideline development, implementation, and measurement science. MedUNAB 2015; 17 (3): 155-162

2. Grinspun D, Lloyd M, Xiao S, Bajnok I. Measuring the Quality of Evidence-Based Nursing Care: NQuIRE Nursing Quality Indicators for Reporting and Evaluation Data-System. MedUNAB 2015; 17(3): 170-175.

3. Grinspun, D.Guías de práctica clínica y entorno laboral basados en la evidencia elaboradas por la Registered Nurses' Association of Ontario (RNAO).EnfermClin 2011; 21(1): 1-2.DOI: 10.1016/j.enfcli.2010.11.002

4. Grinspun, D. Health Policy in Changing Environments. In E. Staples, S. Ray, \& R. Hannon (Eds). Canadian Perspectives on Advanced Nursing Practice: Clinical Practice, Research, Leadership, Consultation and Collaboration Toronto, ON: Canadian Scholar's Press. 2016

5. Registered Nurses' Association of Ontario. (2005). Prevention of Falls and Fall Injuries in the Older Adult. (Revised). Toronto, Canada: Registered Nurses' Association of Ontario 2011. Disponible en: http://rnao.ca/sites/rnao-ca/files/Prevention_of_Falls_ and Fall Injuries in the Older Adult.pdf

6. Registeréd Nurses' Association of Ontario. Assessment and Management of Pressure Injuries for the Interprofessional Team (3rd ed.). Toronto, ON: Registered Nurses' Association of Ontario 2016. Disponible en:http://rnao.ca/sites/rnaoca/files/Pressure_Injuries_BPG.pdf 